\title{
The Cognitive Management of E-Testimony
}

\author{
Saul Traiger
}

\author{
Cognitive Science Program \\ Occidental College \\ Los Angeles, CA 90041U.S.A. \\ traiger@oxy.edu \\ 323-259-2901
}

\begin{abstract}
This paper explores the the justificatory status of etestimony, the electronic transmission of testimony through such electronic media as e-mail, the web, instant messaging, and file-sharing. I argue that e-testimony introduces complexities in justification and cognitive management generally which should be of special interest to epistemologists and cognitive scientists. In contrast to ordinary non-technology mediated testimony, e-testimony is an impoverished stimulus. Users have to assess the epistemic and non-epistemic risks of accessing e-testimony with very little supporting information. This raises the cognitive overhead of such judgments. The
\end{abstract}

\begin{abstract}
paper explores mechanisms for reducing this cognitive overhead and more effectively managing e-testimony, including automated filtering and automated censorship of the incoming e-testimony stream. It is argued that such solutions may not reduce cognitive load, since epistemic responsibility still resides with the individual recipient of e-testimony.
\end{abstract}

Keywords: testimony, e-mail, cognitive management, epistemology

My purpose is to call attention to what I will call e-testimony, testimony transmitted through digital electronic media such as e-mail, the Web, and instant messaging. ${ }^{1}$ I show that e-testimony introduces complexities in justification and cognitive management generally which should be of special interest to epistemologists and cognitive scientists. In this paper, attention is restricted to the e-mail form of etestimony.

Philosophers have long been interested in the question of whether the testimony of others can provide the receiver of testimony with knowledge. ${ }^{2}$ Both the empiricist and rationalist traditions in epistemology have resisted the idea that testimony is a fundamental source of evidence, since it is neither a deliverance of sense nor of reason, but somehow involves both sensory information - the sensory reception of the linguistic output of the testifier - and inferential moves associated with the processing of that output. If knowledge from testimony involves the use of epistemic principles of testimony, what are those principles and from whence do they derive their authority? Are they inductive generalizations from experience, are they a priori truths or derived from such truths, or are they something entirely different? In the $18^{\text {th }}$ century, Hume and Reid attempted to answer these questions, and they did so quite differently. ${ }^{3}$ Then testimony fell off the epistemological map until quite recently.

1 The restriction to digital phenomena is less severe than one might think. The written words and utterances of testifiers is in fact digital, and so such things as phone conversations, even over analog equipment, count as e-testimony.

2 Plato, Charmides, 170d-e. See also (Goldman, 2002) p. 140.

3 Most famously in the Enquiry Concerning Human Understanding Hume (1999) addresses the 
The renewed interest in testimony in philosophy is in part due to the naturalistic turn in epistemology, which can be seen as a return to a Humean science of human nature, now fortified with the experimental and modeling tools of modern cognitive science. If, following and generalizing Quine's famous recommendation, we turn from rational reconstruction to how inquiry actually proceeds, it is startling how many of our beliefs are acquired through testimony. ${ }^{4}$ Epistemologists now look at the role of such things as trust and expertise in the epistemic evaluation of testimony. Describing the formation, organization and transformations in our beliefs and belief systems falls to cognitive science. Describing our doxastic mechanisms can inform the normative, evidential concerns of the epistemologist. Cognitive scientists study such phenomena as perspective taking, belief perseverance, and biases in belief formation, all relevant to the epistemology of testimony.

In a paper extending some of Quine's ideas, Jerry Fodor (1991) argues that epistemic justification should be understood as the task of cognitive management. If our goal is to gain knowledge, then we should adopt strategies in which we are caused to believe that $p$ under those conditions were $p$ is true. A scientist can cognitively position herself to believe that a solution is an acid when litmus paper placed in the solution turns red. A person might position herself to be caused to believe that it will rain tomorrow when rain is forecast in the newspaper. Testimony, then, like sensory evidence, is a type of belief-causing mechanism. The artful inquirer practices cognitive management in order to wind up with true beliefs. Testimony is encountered and evaluated against a background of beliefs. Without the relevant background beliefs about the source of testimony, the properties of the testifier and the medium of testimony transmission, one cannot begin the process of determining the evidential value of the testimony.

Cognitive management of testimony, putting oneself in a position to be caused to believe $p$ when $p$ is true, is a complex process of which the evaluation of a proposition presented by a testifier is just one element. A full treatment of cognitive management and cognitive overhead would investigate the full range of cognitive functions implicated in testimony. Here I will simplify matters significantly by looking only at the range of background beliefs required for the processing of testimony. I will argue that the emergence of etestimony has created additional overhead in terms of the required background beliefs for e-testimony processing. I'll begin with the philosophers' canonical case of receiving testimony, and l'll move toward etestimony.

Consider a case of direct testimony, where you are in unmediated contact with a testifier who communicates testimony through speech. Situated on the platform of at a subway stop in San Francisco, you ask someone if the next train is going to Berkeley, your destination. You are informed that it is. You form the belief that the next train is Berkeley bound and you board it when it arrives. This is belief acquisition through testimony. It's not clear that the receiver of testimony knows, or even has justified belief that the next train is bound for Berkeley.

Now imagine instead that you are alone on the subway platform. A platform sign above announces that the Richmond-bound train will arrive at the platform at 13:10. You consult a posted schedule and see that Berkeley is a stop on the Richmond-bound train. You infer that the next train is Berkeley bound. The mediating technology includes the printed text and platform sign. That the printed word adds cognitive overhead in the evaluation of testimony has long been appreciated. Hume, for example, writes that our beliefs about history depend on "the fidelity of Printers and Copists." When we read history, we make use of our belief that printed copies of a work of history are faithful reproductions of the original work. By relying on this belief, we avoid having to check each copy in the chain of copies from the original manuscript. To take the subway schedule as a piece of relevant evidence, I must believe that the copy of the schedule at which I am looking is a faithful copy of some master schedule.

phenomenon of the widespread belief in miracles. Hume thus takes an important task for the epistemology of testimony to be the explanation of our credulity, the fairly widespread acceptance of testimony where other evidence does not support the belief. Reid (1973), in contrast, argues that people have a God-given propensity to speak the truth, and that our credulity is supported by our justified belief in the existence of that propensity.

${ }^{4}$ See (Quine, 1969). Quine's recommendation is for scientific inquiry, but I take it to generalize to everyday inquiry and knowledge.

${ }^{5}$ See Hume's A Treatise of Human Nature (2000) p. 146. Interestingly, Hume introduces a potential skeptical worry about our reliance of the fidelity of printers and the accuracy of iterated instances of works of history. 
The difference between natural and technology-mediated testimony is the fact that recipients of technology-mediated testimony will employ beliefs about the medium of testimony transmission to cognitively manage testimonial input. Beliefs about testimony transmission will rarely be explicit. But they will have to be accounted for in any procedural epistemology, that is, in any account of how epistemic agents actually draw inferences from testimony. (Pollock, 1998) My claim is that there is a significant increase in cognitive overhead in e-testimony.

Now imagine a morning in cyberspace. While coffee is brewing you fire up your e-mail client. Viewing just the index of messages, containing the sender, the subject, and the date and time, you confront 120 new messages. Each item is a potential piece of testimony. How do you begin to evaluate this torrent? You could look closely at each message or delete all the messages. Devoting 30 seconds to each message will take an hour. If you delete all your messages, you may miss important testimony.

In the first two cases, we confront a single bit of testimony, or at most a bundled set of related messages. In our e-mail example, we have 120 potentially independent messages. Shouldn't we introduce etestimony by considering the evaluation of a single e-mail message? The difficulty is that to access and begin to evaluate the testimony in an individual e-mail message, one must select it from among one's other messages. This distinctive feature is an important source of the testimony management overhead present in e-mail but not always present in other sources of testimony. In our first example, testimony was elicited through a challenge-response cycle. The person in need of information about the subway asks someone who may have the needed information. The response to the challenge either provides that information or it doesn't. If it doesn't, one can attempt to find another potential testifier and repeat the query. In our second example, the prominent subway schedule and signs provide contextual clues to the relevant written testimony. The difference may be summarized as follows: The first subway testimony is synchronous and single-channeled and solicited. The second is asynchronous, single-channeled and solicited The e-mail case under consideration is asynchronous and multi-channeled and may contain both unsolicited and solicited messages.

In addressing the cognitive management issues related to confronting a large volume of e-mail, we need to make explicit some of the factors we might take to be relevant. Time is a factor in the evaluation of testimony. If I were to ask everyone on the platform for directions at once, and had to prioritize responses, sort answers, and epistemically evaluate each one for consistency, relevance, plausibility, etc., I might very well miss the train. Testimony processing time is relative to my interests, practical goals, epistemic goals, and the context.

The more e-mail one receives, the longer it will take to manually process it. Some messages can be read quickly, some may be easy to read and others difficult. Insofar as e-mail received is text, rather than images, video, audio, or other forms of multimedia, the cognitive processing issues for individual messages share the cognitive overhead features of other written testimony. The pre-reading processing of large volume e-mail is of special importance here. When confronted with 120 new messages, what cognitive resources go into digging in and working through the list? Incoming e-mail lists typically contain text fields indicating the sender, the subject, and the time. Compare this to direct, synchronous interaction with testifiers. When one gets subway directions from a "real-time" testifier, there is information about the testifier's appearance, manner, tone of voice, and much more contextual data. Access to more information in the non-technology case may enable a recipient of testimony to more easily manage the information inflow than in e-testimony case.

What follows is a non-exhaustive list of background beliefs and associated cognitive skills and resources which are brought to bear on the retrieval of e-mail testimony. By retrieval, I include the decision to prioritize, to read, and to discard without reading.

- Using the technology While we have shown that technology plays a significant role in a great deal of testimony, in managing one's e-testimony, mastery of the relevant tools is critical. In order to access one's e-mail, one must be able to initialize an e-mail client. This minimal skill presupposes the mastery of many other skills. The use of technology also includes the production of computers, software, networking, and maintenance. Much of this overhead is distributed among the members of organizations, institutions.

- Sorting solicited/ unsolicited e-mail; managing non-received e-mail There are three kinds of email: solicited, unsolicited and e-mail you never get. Solicited e-mail may be a response to an email message you sent, or to a query or request submitted in another medium, e.g. through a conversation, telephone conversation, or instant messaging chat. It may be from an individual or 
an institution. It may be a unique message or a bulk message. Unsolicited mail can be spam, or it could be a message from a long forgotten friend. ${ }^{6}$ Non-received e-mail is e-mail that doesn't make it to your inbox. But you may have evidence that there is such e-mail, and you may have to take steps to recover it. We use cognitive management in order to make these distinctions, and they play a crucial role in our further epistemic and non-epistemic processing of the e-mail torrent.

- Assessing e-testimony The message header of an e-mail message contains a "from:" field as well as "subject:" "date:" and "to:" fields. Solicited and unsolicited messages issue from known correspondents, recognized by name or e-mail address in the "from" field, as well from unrecognized testifiers. Recipients may choose to prioritize e-mail from known senders, on the assumption that known senders will send evidentially salient messages, and that such messages have lower non-epistemic risks. ${ }^{7}$ In much unsolicited bulk e-mail, or e-mail spam, the "from" field is spoofed, and may contain names designed to deceive the recipient. "to:" lines can be spoofed as well to make it appear that the sender knows the recipient. Information about the content of the message, either in the subject line, or elsewhere, may influence our judgments about the source of the testimony. Deceptive subject line content may present the appearance that the message is a solicited message, or is from a trusted institution or individual.

The use of selective channels in e-mail and other technology media can serve as an aid to evidential assessment. An example is the use of private short-wave radio channels by public safety personnel. By using a channel-secured radio device, all transmissions are from trusted, expert sources. The medium provides an evidential stamp of approval. The use of the medium provides a reduction in cognitive load. The recipient of testimony through such a medium can reduce some of her effort in evidential evaluation. E-mail is at least initially multi-channeled. ${ }^{8}$

- Assessing non-epistemic risks If you ask the wrong person for directions in a dark alley, you may loose more than knowledge. Thus an aspect of cognitive management even where technology is not involved in the transmission of testimony is the assessment of non-epistemic risks and benefits. Testimony through e-mail can include any digitally encoded information, including video, audio, and executable program files. Retrieving an e-mail message can cause significant harm to the recipient in the form of a virus, a Trojan, or a denial of service attack. This kind of overhead might be treated a subcategory of use of technology, but it fits more broadly under risk assessment for testimony generally. The overhead under discussion is not the cost incurred when such risks materialize, but rather the cognitive work of assessing the risks associated with such events. $^{9}$

- Assessing evidential content Assessing the evidential worth of testimony through e-mail includes determining authorship, as well as looking at the content of the subject field and ultimately the body of the message. Unsolicited e-mail may be particularly difficult to assess, since much of it is intentionally deceptive in both content delivered and the purported identity of the sender. The message may also contain content that is embedded, either in the markup code of the message or in attachments. So one simply may not be able to assess the content of the message through ordinary observation.

These are tasks for individuals managing the inflow of e-mail. Most treatments of the problems with e-mail treat spam and its overall economic impact. In contrast I have made an effort to list some of the background beliefs and associated skills which are required for carrying out these tasks. ${ }^{10}$

${ }^{6}$ What counts as an unsolicited e-mail message, or unsolicited communication of any other kind is a complex matter, and we cannot give it its due here. In normal circumstances, some individuals might have an open channel to others which does not require an opening or renewed invitation or query for their communications to count as solicited.

7 This assumption is notoriously problematic, due the fact that spam can be sent from a user's machine or e-mail address without permission or knowledge.

${ }^{8}$ E-mail can be single-channeled. For example, one can filter all e-mail from a specific sender to a special mailbox.

9 Dreyfus (1999) argues that risks via "telepresence," which includes e-mail, are minimized by users, compared to their direct experiential interactions with risk. The research Dreyfus cites in support of this appears to predate the now widespread risks in e-mail and the Web.

10 One limitation of this brief treatment is that it does not take into account the broad epistemic phenomena, including the consequences of current e-testimony structure in terms of lost epistemic 
Goldman is one of the few epistemologists to devote attention to the role of digital technology in the acquisition of knowledge. He sets out a form of epistemic consequentialism. If the goal of inquiry is truth, then our social epistemic practices generally, and our technology-implicated practices in particular, should be evaluated relative to the fulfillment of that goal. So good practices are those which yield true belief, and the more the better. Bad practices or conditions are those which don't yield truth. The epistemic "goodness" and "badness" are assessed in terms of quantity of knowledge for Goldman.

We read our e-mail in order to gain knowledge of various sorts. Yet the vast majority of e-mail sent is not sent by those who share our epistemic goals. When we check our e-mail, our cognitive strategies must be mindful of this, and must enable us to negotiate the e-mail mine-field. Picking out that tiny bit of potentially knowledge-increasing testimony from the torrent of incoming e-mail requires careful cognitive management. The wrong cognitive strategy can have bad consequences, both epistemic and nonepistemic. The point was made long ago by Hume. He wondered how belief in miracles could be so widely believed and propagated, since a miracle is something for which there can be no evidence from observed regularities. The answer is that there are all sorts of non-epistemic factors which play a role in the transmission of testimony, such as the pleasure testifiers get from reporting something new and marvelous. More recently, Dan Sperber (2001) suggests that the transmission of truth is just one among a host of goals which can be served through the transmission of testimony. Testimony may be transmitted in order to exert power of the recipient of testimony, to acquire resources from the recipient, to inflict harm to the individual and to the political, social, or other group of which the recipient is a member. One must distinguish incoming e-mail to messages which are directed towards one's epistemic goals from ones which are not before the process of epistemic evaluation of testimony can begin. ${ }^{11}$

We need both a descriptive account of the user's cognitive load present in the management of e-mail, and a normative theory of how best to bring cognitive resources to bear in the evaluation of the evidential worth of e-mail messages. There could be a normative theory about how to achieve other ends through email, e.g. a theory of how to make money through e-mail. Indeed such theories abound. ${ }^{12}$

To what use should we put such descriptive and normative theories? There are numerous applications. First, understanding the cognitive load in the use of e-mail is a project for human/computer interface ( $\mathrm{CHI})$ engineers and theoreticians. Much of the work in this area concentrates on the "use of technology" cognitive skills, with particular emphasis of the level of difficulty experienced in processing e-mail relative to the mastery of e-mail client and related operating system software. (Lantz, 2003) The observations above suggest that cognitive processing of e-mail has changed as a function of the changing role of email, particularly the explosion in unsolicited commercial e-mail. A longitudinal study by Lantz explores how the use of e-mail changes over time, including the change in the number of e-mail messages received and the facility of the user in processing the flow of e-mail. This study took place within a corporate intranet, before the explosion of spam. This methodology could be used to study the phenomena described above. Whittaker and Sidner (1996) study what they call "email overload" in the context of personal information management. They argue that a source of e-mail overload is that e-mail is now used for a much wider range of personal information management tasks than simple interpersonal communication. E-mail is used for document storage, task prioritization, calendaring, and other functions. $\mathrm{CHI}$ methodology has the resources to address these issues and to begin to take into account the changes in the social epistemology of e-testimony. Of course one of the mail goals is to create tools which offload the cognitive overhead.

Can cognitive overhead be reduced by offloading the cognitive burden to software? E-mail filters, for example, and other technology attempt to reduce the number of messages which require user evaluation. (Goodman, et. al., 2005) Manual filtering requires the user to specify strings of text which sort messages into specified mailboxes, label the messages, or perform other actions, including message deletion. While such filters can be helpful, proper installation requires significant cognitive investment, and improperly set

opportunities.

11 Goldman's consequentialism does not specify how the "amount" of true belief is to be measured. If someone believes $\{p, q, r\}$ do they believe more than if they belief $\{s, t\}$ ? That of course depends on the content of the propositions. Without a measure of the content of propositions, Goldman's epistemic consequentialism is radically incomplete. And attempts to provide such an account, e.g. Popper's (1979) have been notoriously problematic.

${ }^{12}$ McWilliams (2005) describes the widespread practice among e-mail spammers of selling guides to the art of spamming. 
filters can lead to the problem of false positives - legitimate messages which are treated as spam and deleted. It's not clear that such manual techniques reduce cognitive overhead.

The limitations of manual filtering has led to the most widely used current approach, automated filtering. Typically a probability that the message is spam is assigned by analyzing strings in messages, using machine learning. The filters segregate or delete e-mail before it gets to the user when the probability reaches a threshold value. Filters can search the content of entire messages, not just the headers, and take appropriate (or inappropriate) actions in advance of the user's access to that content. Building a discriminatory ability into the software might be viewed as helping to remedy the poverty of the stimulus problem. Automated filters can present messages to users with the additional information that a message has survived the filters and hence is worthy of further epistemic evaluation. The filtering mechanism thus serves as additional testimony, just as various aspects of the environment can serve as supporting evidence in the case of direct testimony. ${ }^{13}$

13 Haugeland (1998) provides an useful analysis of the fluidity of information-possession from the individual to objects and individuals in the world. 


\section{References}

Androutsopoulos, Ion, et. al. (2000) "An Evaluation of Naïve Bayesian Anti-Spam Filtering, Proceedings of the Workshop on Machine Learning in the New Information Age, G. Potamias, et. al., (eds.) $11^{\text {th }}$ European Conference on Machine Learning, Barcelona, Spain, pp. 9-17.

Anscombe, E. (1981) "Hume and Julius Caesar" in From Parmenides to Wittgenstein: Collected Philosophical Papers Volume 1 Oxford, Basil Blackwell.

Coady, C. A .J. (1992) Testimony: A Philosophical Study. Oxford: Clarendon Press.

Dreyfus, Hubert, (1999) “Tele-epistemology: Descartes' Last Stand” in Goldberg, Ken, ed., TheRobot in the Garden: Telerootics and Telepistemology in the Age of the Internet (Cambridge, MA: MIT Press) pp. 48-63.

Fodor, Jerry (1991) “The Dogma That Didn't Bark” Mind, vol. 100, pp. 201-220.

Goldman, Alvin (1991) "Epistemic Paternalism: Communication Control in Law and Society", The Journal of Philosophy LXXXVIII, 3 pp. 113-131.

Goldman, Alvin (1999) Knowledge in a Social World, Oxford: Oxford University Press.

Goldman, Alvin (2002) Pathways to Knowledge: Public and Private, (Oxford: Oxford University Press

Goodman, Joshua, et. al. (2005) "Stopping Spam" Scientific American.

Graham, Paul (2003) "Will Filters Kill Spam?" Computer Security Journal January, 2003; reprinted as http://www.paulgraham.com/wfks.html.

Haugeland, John, (1998) "Mind Emboddied and Embedded" in Haugeland, John, Having Thought (Cambridge, MA: Harvard University Press, pp. 207-240.

Hume, David (1999) An Enquiry concerning Human Understanding, Tom L. Beauchamp, ed. Oxford: Oxford University Press. Hume, David, (2000) A Treatise of Human Nature, David Fate Norton, Mary J. Norton, eds. Oxford: Oxford University Press. Judge, Paul; (2003) "The State of the Spam Problem", Educause, pp. 60-61.

Lantz, A.(2003) "Does the Use of E-Mail Change Over Time?" International Journal of Human-Computer Interaction, 15(3), 419-431.

McDowell, Ashley (2002) "Trust and information: the role of trust in the social epistemology of information science" Social Epistemology 16 No: 1.

McWilliams, Brian (2005) Spam Kings, Sebastopol, CA: O'Reilly \& Associates.

Pollock, John L. (1998) "Procedural Epistemology" in The Digital Phoenix: How Computers are Changing Philosophy Oxford: Blackwell.

Popper, Karl R.(1979) Objective Knowledge Oxford: Oxford University Press.

Quine, W.V.O. (1969) “Epistemology Naturalized” in Ontological Relativity and Other Essays NY: Columbia University Press, pp. 6990.

Reid, Thomas, (1973) Inquiry and Essays, Ronald Beanblossom and Keith Lehrer, eds. Indianapolis: Hackett.

Schmitt, Frederick (1987) "Justification, Sociality, and Autonomy", Synthese 73.

Welbourne, Michael (2001) Knowledge, Chesham: Acumen.

Whittaker, S., \& Sidner, C. (1996) "Email overload: Exploring personal information management of email” In M. J. Tauber (Ed.),

Proceedings of $\mathrm{CHI}$ '96 (pp. 276-283).New York: ACM. 